\title{
Sarcoma de Kaposi associado à SIDA: relato de caso
}

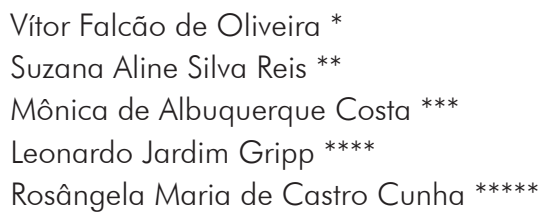

\section{RESUMO}

Sarcoma de Kaposi (SK) é uma neoplasia angioproliferativa multifocal de etiologia viral e patogênese multifatorial, com a presença de múltiplos nódulos hiperpigmentados e elevados, podendo acometer pele e tecido subcutâneo. Relatamos paciente infectado pelo Vírus da Imunodeficiência Humana (HIV) há 10 anos, de transmissão ignorada, com lesões em membros inferiores (MMII) de cor violácea, nodulares, não pruriginosas, indolores; diagnosticadas como SK por estudo anatomopatológico e imunohistoquímico. Realizado screening para doença disseminada com endoscopia digestiva alta (EDA), radiografias de tórax e ósseas nos membros acometidos, ultrassonografia (USG) de abdome total e a videolaringoscopia, que não demostraram lesão inicial. Paciente durante a internação teve avaliação dos Serviços de Proctologia, Urologia e Otorrinolaringologia. A terapêutica empregada com base na introdução da terapia antirretroviral (TARV) teve sucesso na regressão das lesões em MMII, sem necessidade de quimioterapia ou radioterapia.

\section{Palavras-chave: Sarcoma de Kaposi. Hospedeiro Imunocomprometido. Síndrome da Imunodeficiência Adquirida.}

\section{INTRODUÇÃO}

Sarcoma de Kaposi (SK) é uma doença angioproliferativa multifocal de etiologia viral e patogênese multifatorial (KAPOSI, 1972). Foi descrito pela primeira vez em 1872 pelo dermatologista Moritz Kaposi como "sarcoma hiperpigmentado, múltiplo e idiopático da pele" (KAPOSI, 1982). Desde então, o SK tem sido definido como uma neoplasia maligna de vasos sanguíneos ou linfáticos, com a presença de múltiplos nódulos hiperpigmentados e elevados, podendo acometer pele e tecido subcutâneo, mais frequentemente em membros inferiores, cabeça e pescoço (PANTANOWITZ; DEZUBE, 2008). Além disso, pode cursar com comprometimento visceral amplamente disseminado (LUNDGREN et al., 1997).

A apresentação clínica do SK pode ser classificada em quatro formas distintas: clássico, endêmico, iatrogênico (associado à imunossupressão) e epidêmico (SIDA-associado). Essa patologia é uma malignidade associada à infecção pelo o vírus HIV, sendo a classificação SIDA-associado a forma mais agressiva (LEÃO et al., 1999).

Com a progressão da epidemia do HIV, a disfunção do sistema imune devido ao vírus predispõe o desenvolvimento de doenças oportunistas e neoplasias incomuns. O SK foi encontrado quase exclusivamente entre homens homossexuais, em que os dados epidemiológicos indicaram alta incidência da doença entre pessoas de maior risco de infecções sexualmente transmissíveis (HERMANS et al., 1996).

$\mathrm{Na}$ década de 90 , o agente infeccioso causal foi atribuído ao Herpes Vírus Humano 8 (HHV-8). Tal associação não é suficiente para desenvolver a neoplasia, necessitando-se, então, de fatores genéticos e imunológicos predisponentes (FIFE; BOWER, 1996; STEBBING; PORTSMOUTH; BOWER, 2003).

O objetivo deste relato, portanto, foi apresentar um caso de SK associado a SIDA e realizar breve revisão de literatura.

\footnotetext{
* Universidade Federal de Juiz de Fora, Faculdade de Medicina - Juiz de Fora, MG. E-mail: vitorfalcaodeoliveira@gmail.com

** Universidade Federal de Juiz de Fora, Faculdade de Medicina - Juiz de Fora, MG.

*** Hospital Universitário da Universidade Federal de Juiz de Fora, Serviço de Dermatologia - Juiz de Fora, MG.

**** Hospital Universitário da Universidade Federal de Juiz de Fora, Serviço de Patologia - Juiz de Fora, MG.

***** Universidade Federal de Juiz de Fora, Faculdade de Medicina, Departamento de Clínica Médica - Juiz de Fora, MG.
} 


\section{Relato de CASO}

Paciente MA, sexo masculino, 48 anos, solteiro, natural do Rio de Janeiro e residente em Juiz de Fora, admitido no serviço de Infectologia do Hospital Universitário da Universidade Federal de Juiz de Fora (HU-UFJF) queixando-se de aparecimento de lesões em membros inferiores (MMII) de cor violácea, nodulares, não pruriginosas, indolores, com aparecimento há 1 ano, bilateral em pernas e unilateral em coxa esquerda. Paciente também relatava lesões verrucosas em corpo do pênis e ânus há 2 anos, com diagnóstico clínico prévio de lesão por papiloma vírus humano (HPV).

Foi infectado pelo Vírus da Imunodeficiência Humana (HIV) há 10 anos, de transmissão ignorada. Foram solicitadas carga viral e contagem de CD4, cujos valores foram, respectivamente, 4.043 cópias e 118 células $/ \mathrm{mm}^{3}$. Além disso, o paciente era virgem de tratamento até 01 semana antes da internação hospitalar, sendo prescrito a terapia antirretroviral (TARV) com Lopinavir/Ritonavir (IP) associado Tenofovir (TDF) e Lamivudina, modificado posteriormente devido à piora da função renal secundária à nefrotoxicidade do TDF. Então, foi prescrito Zidovudina e Lamivudina em substituição ao esquema anterior, mantendo o IP. Paciente foi submetido à profilaxias para pneumocistose e toxoplasmose.

Ao exame físico, encontrava-se estável hemodinamicamente, com aparelhos cardiovascular, respiratório e abdome sem alterações. Ao exame dos MMII, presença de lesões nodulares purpúricas, sem edema e com panturrilhas livres (Figura 1):

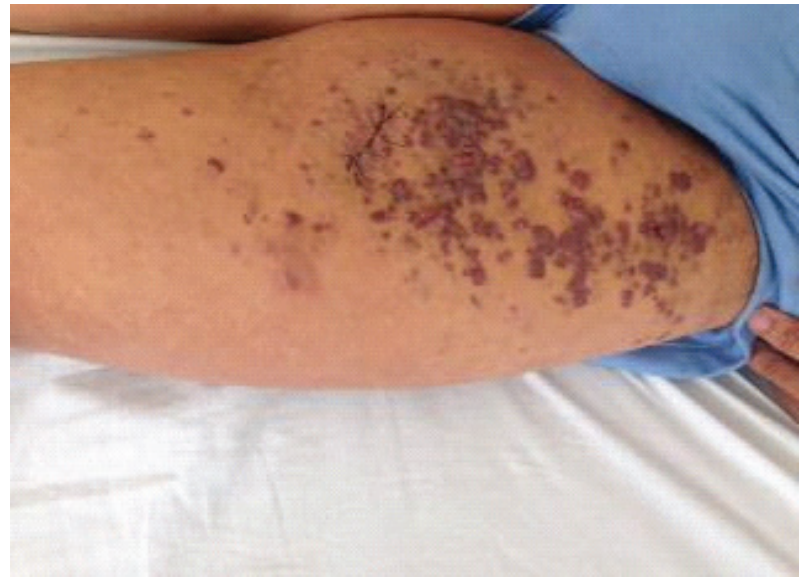

Figura 1: Imagem das lesões nodulares purpúricas em MMII.

Fonte: Os autores (2017).

Foram realizados exames laboratoriais na admissão que identificaram função renal e hepática dentro do limite da normalidade, e hemograma sem alterações significativas.

Paciente realizou biópsia das lesões em perna esquerda, com material enviado para estudo anatomopatológico e microbiológico, que identificaram como negativas a pesquisa para bacilo álcool-ácido resistente (BAAR), a pesquisa direta de fungos, a cultura para bactérias, micobactérias, fungos e GRAM. No exame anatomopatológico, foi identificado nódulo bem circunscrito composto por células fusiformes com citoplasma eosinofílico em arranjo estoriforme, entremeadas por fendas vasculares com hemácias extravasadas (Figuras 2, 3 e 4), achados histopatológicos compatíveis com Sarcoma de Kaposi. Além disso, foi realizada a imunohistoquímica da biópsia que revelou expressão para marcadores endoteliais, CD31 e CD34, além da expressão nuclear para HHV-8, confirmando o diagnóstico de SK (Figura 5).

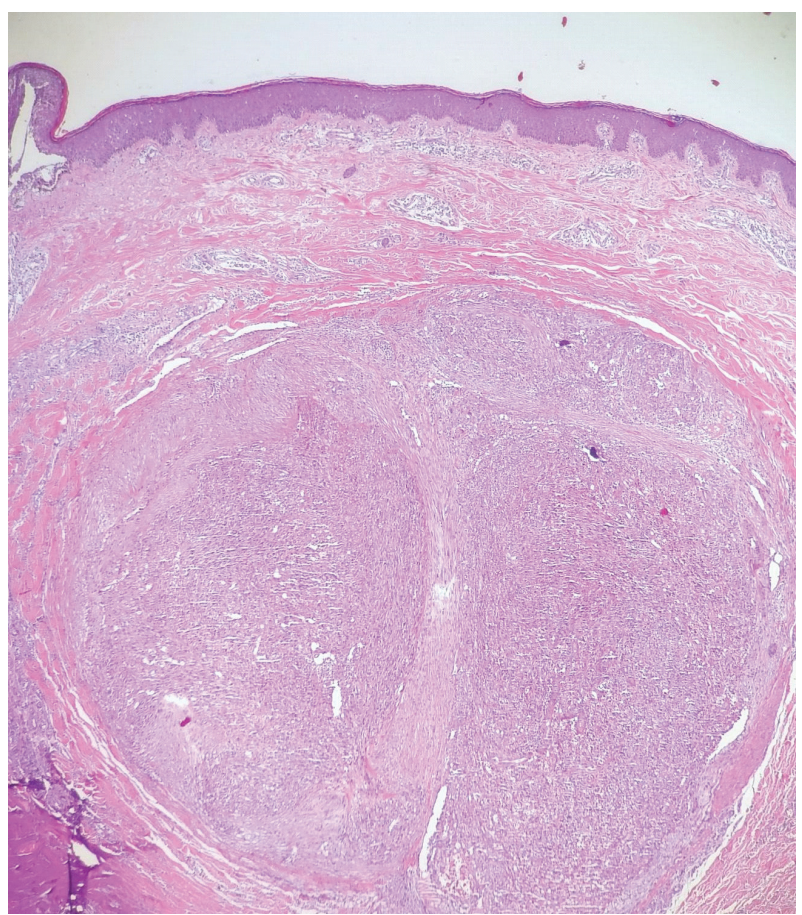

Figura 2: Imagem anatomopatológica de nódulo dérmico bem delimitado, com vasos ectásicos periféricos e fendas centrais em coloração de Hematoxilina-eosina, 40x.

Fonte: Os autores (2017)

Foi realizada endoscopia digestiva alta (EDA) para pesquisa de doença disseminada, onde foram identificados os seguintes achados: gastrite endoscópica erosiva elevada leve de antro, dois pólipos em fundos gástricos com estudo anatomopatológico laudado como pólipo hiperplásico/inflamatório. As radiografias de tórax e ósseas nos membros acometidos, a ultrassonografia (USG) de abdome total e a videolaringoscopia não demostraram lesão inicial. Enquanto no Ecodoppler venoso dos MMII, foram identificados múltiplos linfonodos proeminentes e edema do tecido subcutâneo na face ântero-medial da coxa. 


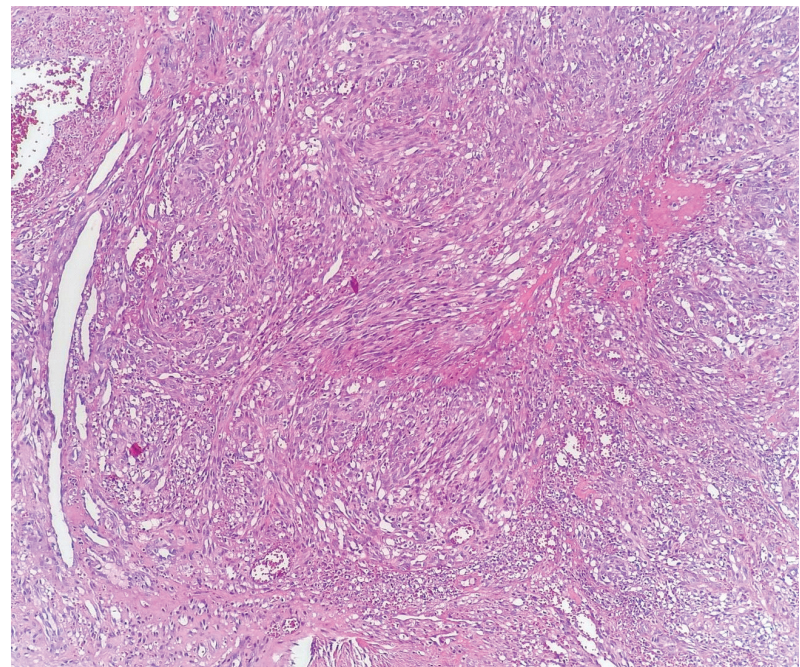

Figura 3: Imagem anatomopatológica de células fusiformes em arranjo estoriforme, fendas vasculares irregulares em coloração de Hematoxilinaeosina, 100x.

Fonte: Os autores (2017).

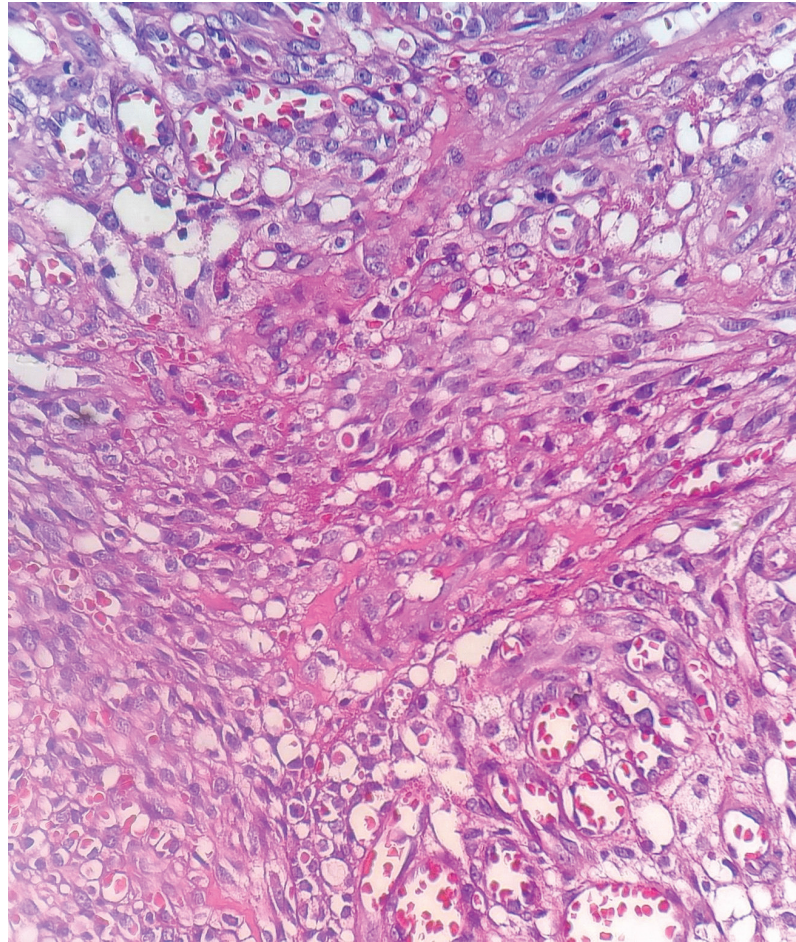

Figura 4: Imagem anatomopatológica de células fusiformes com citoplasma eosinofílico, hemácias extravasadas em coloração de hematoxilina-eosina, 400x.

Fonte: Os autores (2017).

Paciente:

Idade: 48 anos Sexo: Masculino

|IIIIIIII||||||

História Clinica: Biópsia de lesão em perna esquerda.

nacebido am

Material Recebido: Bloco(s) de parafina (2) e Lâmina(s) (2) rotulado(s) como: 16/3792-3793
Procedència: Juiz de Fora (MG)

Estudo imuno-histoquimica: Após desparafinaçăo e tratamento dos tecidos com soluçốs próprias para recuperaçấo de epitopes, os ceries histologicon

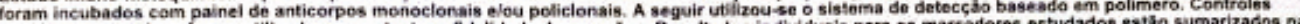

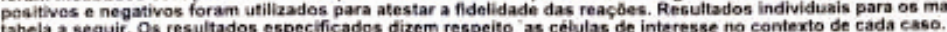

\begin{tabular}{|c|c|c|}
\hline Anticarpos & Clone & Resultado \\
\hline - HHV-8 & $L N-53$ & Pasitive \\
\hline - CD31 - moléeala de adesào PECAM-1 (etlulas endoteliais) & JChou & Pasitive \\
\hline - CD34 - antigeno de células hematopoéticas e pericitos & QBEnd 10 & Pusitive \\
\hline - Desmina (filamento intermediario cellala muscular) & D33 & Negativo \\
\hline - Citoceratinas de $40,48,50$ e $50,6 \mathrm{kDa}$ & AE $1 / A E_{3}$ & Negatico \\
\hline - Proteina S-160 & Policlonal & Negativo \\
\hline
\end{tabular}

\section{Conclusão:}

LESÃO DE PERNA ESQUERDA: SARCOMA DE KAPOSI (WHO, 2013). VER COMENTÁRIO.

\section{Comentário:}

Trata-se de lesto fusocelular acometendo derme,com crescimento em padräo nodular, exibindo fendas vasculares, glóbulos hialinos, extravasamento de hemácias e ocasionais figuras de mitose, 0 estudo imuno-histoquimico revelou expressão para marcadores endoteliais, CD31 (foto 2) e CD34, além de expressào nuclear para HHV-8 (foto 3). 0 conjunto dos achados é diagnóstico de SARCOMA DE KAPOSI.

Bibliografia:

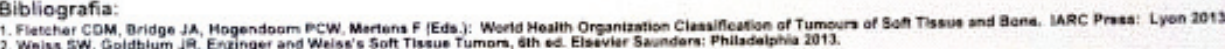
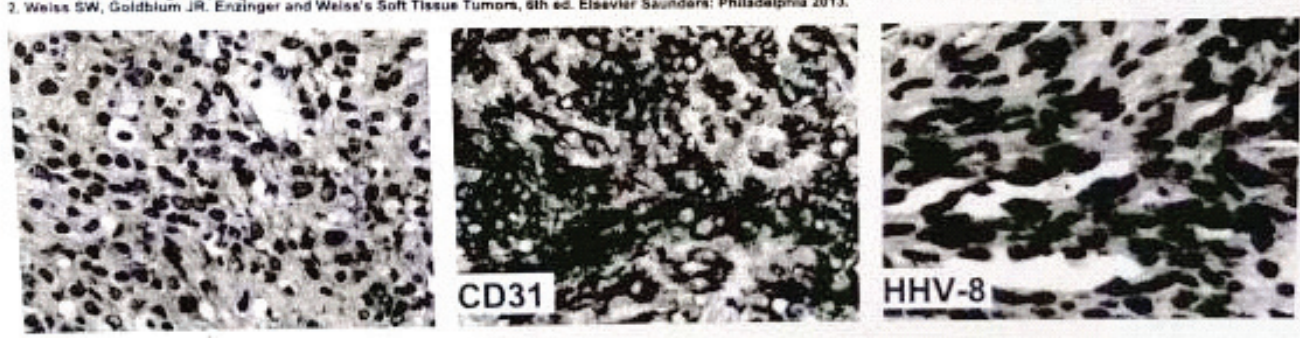

Figura 5: Imagem do laudo do estudo imunohistoquímico.

Fonte: Os autores (2017). 
Ademais, as sorologias para vírus B, C e anti-HTLV foram negativas. Já as sorologias para toxoplasmose $\operatorname{IgG}$, citomegalovirose (CMV) IgG, teste treponêmico e VDRL foram reagentes.

Paciente durante a internação teve avaliação dos Serviços de Proctologia, Urologia e Otorrinolaringologia. Os serviços de Urologia e Proctologia orientaram o uso de podofilina tópica para aplicar nas lesões condilomatosas e acompanhamento ambulatorial. Além disso, foi orientado a realização de colonoscopia ambulatorial após o tratamento das lesões anais. Paciente foi encaminhado para o serviço de oncologia clínica que manteve TARV e seguimento. Após iniciar a TARV, notou-se regressão das lesões em MMII.

Relato de caso autorizado pelo paciente e aprovado no Comitê de Ética em Pesquisa do Hospital Universitário da Universidade Federal de Juiz de Fora (CEP HU-UFJF).

\section{Discussão}

O diagnóstico diferencial do SK deve incluir doenças como, linfangioendotelioma benigno, acroangiodermatite, angiosarcoma cutâneo, angiolipoma, histiocitoma fibroso aneurismático, angiomatose bacilar, linfoma cutâneo e hemangioma hemosiderótico (CHOR; SANTA CRUZ, 1992).

Em relação à histopatologia, as lesões apresentam três fases: as fases de mácula e placa, que consistem em proliferação de vasos dérmicos com atipia endotelial mínima, circundados por infiltrado inflamatório linfoplasmocitário, com hemácias extravasadas ou depósitos de hemossiderina. À medida que a lesão evolui, inicia-se a fase nodular, com formação de nódulos dérmicos bem delimitados constituídos por células fusiformes com citoplasma eosinofílico, com numerosas mitoses e fendas vasculares irregulares, sem revestimento endotelial, espalhadas pelo nódulo. A imunohistoquímica das células fusiformes é geralmente CD34 e CD31 positiva, indicando origem endotelial, e LNA-1 para identificação de infecção pelo Herpes Vírus Humano 8 também é positiva (RADU; PANTANOWITZ, 2013).

O SK apresenta-se clinicamente como múltiplos nódulos vascularizados, podendo também acometer pele e outros órgãos. Portanto, o padrão dessa patologia é variável, com manifestação apenas composta por nódulos cutâneos, sem envolvimento visceral, ou apresentação fulminante, com lesões cutâneas extensas e acometimento visceral. Assim, o SK pode surgir em mucosa oral, linfonodos ou em vísceras, sem lesões em pele, sendo necessário investigar doença disseminada (SCHWARTZ, 2004).
A maioria das lesões gastrointestinais apresenta aspecto clínico silencioso, sendo algumas com evidência de doença visceral, porém, sem doença cutânea. Aproximadamente $50 \%$ dos pacientes têm lesões maculo-papulares, pólipos ou nódulos umbilicados em um ou mais sítios ao longo do tubo digestivo. Para avaliar o envolvimento visceral, podese utilizar radiografia (enema opaco), endoscopia e colonoscopia. Devido à localização submucosa do tumor, a biópsia torna-se um exame menos sensível para o diagnóstico (FRIEDMAN; WRIGHT; ALTMAN, 1985).

As lesões do SK pulmonar podem localizar-se em: parênquima pulmonar, árvore brônquica ou pleura. As repercussões clínicas, consequentemente, são obstrução brônquica, insuficiência respiratória e derrame pleural (LUNDGREN et al., 1997). Então, os testes de função pulmonar também são sensíveis na detecção de anormalidades ocasionadas pela neoplasia. Além da clínica, a radiografia e a broncoscopia podem auxiliar no diagnóstico. Apesar do SK endobrônquico ser visualizado na broncoscopia como lesões ligeiramente sobrelevadas e de coloração vermelho-cereja, a biópsia brônquica não demonstra alterações. As alterações radiográficas sugestivas de SK, por sua vez, incluem infiltrados intersticiais bilateral e/ou alveolares, muitas vezes com nodularidade mal definida. Desse modo, infiltrados nodulares com ou sem derrame pleural são sugestivos de envolvimento pulmonar pelo SK em pacientes com SIDA (MEDURI et al., 1986).

O SK pode também envolver regiões de mucosa, como gengiva, língua, palato, tonsilas e faringe, com possibilidade de interferir na deglutição (FICARRA et al., 1988). Por isso, foi realizada videolaringoscopia, porém, não foram identificadas lesões nos compartimentos do andar supra-glótico, mucosa laríngea, andar glótico e infra-glótico, com exame dentro do limite da normalidade.

Além disso, há casos descritos na literatura de SK acometendo outros órgãos internos, a exemplo do baço, fígado, ossos e medula óssea, sendo extremamente raro no Sistema Nervoso Central (LUNDGREN et al., 1997). O USG de abdome total demonstrou fígado, vesícula biliar, baço, pâncreas e rins com contornos regulares, textura homogênea e tamanho normal, sem demais alterações em vias biliares, aorta e bexiga. Desse modo, o estadiamento do SK possui como objetivo estabelecer o prognóstico e orientar a terapêutica (FONSECA et al., 1999).

É inegável que a abordagem do SK e os métodos de tratamento estão em evolução e em constantes mudanças, principalmente diante das modificações do HIV. O curso clínico, a escolha terapêutica e o 
prognóstico são fortemente influenciados pelo padrão tumoral, grau de disfunção imune e presença de infecções oportunistas. Dessa forma, é fundamental que o tratamento seja individualizado (PDQ, 2015).

O grande objetivo do tratamento, além da cura efetiva do paciente, é aliviar os sintomas, prevenir a progressão da doença, reduzir o tamanho do tumor e envolvimento de possíveis órgãos afetados. Nesse aspecto, pacientes que não fazem uso da terapia antirretroviral devem iniciá-la ao diagnóstico de SK, uma vez que o surgimento da neoplasia está associado à imunodeficiência. Sendo assim, a recuperação do estado imunológico de pacientes infectados ou não pelo HIV trouxe regressão parcial ou total das lesões de SK (FONSECA et al., 1999).

No SK epidêmico associado à SIDA, a quimioterapia é o padrão ouro no tratamento, utilizando-se agentes únicos ou combinações. A doxorrubicina lipossomal é a terapia de primeira linha para a doença e, em caso de falha terapêtica, paclitaxel (taxol) é empregado atualmente como segunda linha (KRELL \& STEBBING, 2013). Foi observado que a doxorrubicina lipossomal ocasiona benefícios clínicos, tais como diminuição das lesões, com melhora da coloração, dor e edema associados à lesão (NORTHFELT et al., 1997).

Todavia, diante da terapia imunossupressora, aumenta-se o risco de outras infecções oportunistas, sendo que quanto mais agressivo for o esquema quimioterápico, menor é a tolerância hematológica do paciente e, por isso, estudos sugerem a adoção preferencial de antraciclinas lipossômicas. Nesses casos, os efeitos adversos geralmente são mais leves, sendo alopécia e neuropatias incomuns. O paclitaxel também é bem tolerado, porém, apresenta maior taxa de efeitos colaterais e demanda maior tempo para infusão, em comparação à doxorrubicina (GILL, 1999).

Em lesões cutâneas generalizadas, a radioterapia é uma opção de tratamento, a qual também é recomendada para paliação da dor (KIROVA et al., 1998). Além disso, curetagem, crioterapia ou excisões cirúrgicas também podem ser utilizadas em áreas limitadas (DI LORENZO et al., 2007).

A eletroquimioterapia é a mais recente modalidade usada para o tratamento de neoplasias cutâneas ou subcutâneas, a qual permite a maior biodisponibilidade de drogas na região. (DI MONTA et al., 2014). Agentes biológicos também estão sendo aplicados no tratamento, mostrando bons resultados na regressão das lesões (KOON et al., 2014).

Estudos recentes descrevem um tipo de SK mais agressivo, associado à Síndrome de Reconstituição Imune (SRI), definida por progressivo declínio das condições clínicas consequentes à recuperação do sistema imunitário. Com o advento da maior aderência e distribuição do tratamento antirretroviral, demonstraram que a introdução da terapia em infecções avançadas pelo HIV pode estar associada a uma reativação de doenças silenciosa, exemplificadas principalmente por associação ao Mycobacterium tuberculosis e Cryptococcus neoformans. Dessa forma, pode ser uma doença sugestiva de SRI quando associada a um significante aumento da contagem de CD4 no momento do diagnóstico de SK (BOWER et al., 2005).

\section{Conclusão}

O diagnóstico clínico e histopatológico da lesão, assim como a realização de exames complementares, foi feito para investigação de doença disseminada, não evidenciando demais acometimentos, somente lesões cutâneas. Por fim, a terapêutica empregada com base na introdução da TARV teve sucesso na regressão das lesões em MMII, sem necessidade de quimioterapia ou radioterapia. 


\title{
Kaposis's sarcoma associated with AIDS: case report
}

\begin{abstract}
Kaposi's Sarcoma (KS) is a multifocal angioproliferative neoplasm of viral etiology and multifactorial pathogenesis, with the presence of multiple hyperpigmented and elevated nodules, which can affect skin and subcutaneous tissue. We reported a patient who was infected by the Human Immunodeficiency Virus (HIV) 10 years ago and had unknown the way of transmission, with lesions in the lower limbs (LMW) of violet color, nodular, not pruritic, painless; diagnosed as Kaposi's sarcoma by anatomopathological and immunohistochemical study. Screening for disseminated disease with upper digestive endoscopy (UDE), chest and bony radiographs on affected limbs, total abdomen ultrasonography (USG) and videolaryngoscopy, which did not show an initial lesion. Patient had been evaluated by Proctology, Urology and Otorhinolaryngology medical specialties. The treatment was based on the introduction of antiretroviral therapy which allowed in the regression of lesions and effectiveness without the need for chemotherapy or radiotherapy.
\end{abstract}

Keywords: Sarcoma, Kaposi. Immunocompromised Host. Acquired Immunodeficiency Syndrome.

\section{REFERÊNCIAS}

BOWER, M. et al. Immune reconstitution inflammatory syndrome associated with Kaposi's sarcoma. Journal of Clinical Oncology, v. 23, n. 22, p. 5224-5228, aug. 2005.

CHOR, P. J.; SANTA CRUZ, D. J. Kaposi's sarcoma. Journal of Cutaneous Pathology, v. 19, n. 1, p. 6-20, 1992.

DI LORENZO, G. et al. Management of AIDS-related Kaposi's sarcoma. The Lancet Oncology, v. 8, n. 2, p. 167-176, feb. 2007.

DI MONTA, G. et al. Electrochemotherapy as "new standard of care" treatment for cutaneous Kaposi's sarcoma. European Journal of Surgical Oncology (EJSO), v. 40, n. 1, p. 61-66, jan. 2014

FICARRA, G. et al. Kaposi's sarcoma of the oral cavity: a study of 134 patients with a review of the pathogenesis, epidemiology, clinical aspects, and treatment. Oral Surgery, Oral Medicine, Oral Pathology, v. 66, n. 5, p. 543-550, nov. 1988.

FIFE, K.; BOWER, M. Recent insights into the pathogenesis of Kaposi's sarcoma. British Journal of Cancer, v. 73, n. 11, p. 1317, jun. 1996.

FONSECA, B. A. L.; BOLLELA, V. R.; NETO, R. D. J. P. Sarcoma de Kaposi e síndrome da imunodeficiência adquirida: características desta associação, incluindo novos conceitos sobre patogênese e tratamento. Medicina, Ribeirão Preto, v. 32, n. 1, p. 26-39, jan./mar. 1999.

FRIEDMAN, S. L.; WRIGHT, T. L.; ALTMAN, D. F. Gastrointestinal Kaposi's sarcoma in patients with acquired immunodeficiency syndrome: endoscopic and autopsy findings. Gastroenterology, v. 89, n. 1, p. 102-108, jul. 1985.

GILL, P. S. et al. Randomized phase III trial of liposomal daunorubicin versus doxorubicin, bleomycin, and vincristine in AIDS-related Kaposi's sarcoma. Journal of Clinical Oncology, v. 14, n. 8, p. 2353-2364, aug. 1996.
HERMANS, P. et al. Epidemiology of AIDS-related Kaposi's sarcoma in Europe over 10 years. AIDS, v. 10, n. 8, p. 911-918, jul. 1996.

KAPOSI, M. Idiopathisches multiples pigmentsarkom der haut. Archives of Dermatology and Syphilology, v. 4, p. 265273, 1872.

KAPOSI, M. Idiopathic multiple pigmented sarcoma of the skin. CA: A Cancer Journal for Clinicians, v. 32, n. 6, p. 342347, 1982.

KIROUA, Y. M. et al. Radiotherapy in the management of epidemic Kaposi's sarcoma: a retrospective study of 643 cases. Radiotherapy and Oncology, v. 46, n. 1, p. 19-22, jan. 1998.

KOON, H. B. et al. Phase II trial of imatinib in AIDS-associated Kaposi's sarcoma: AIDS Malignancy Consortium Protocol 042. Journal of Clinical Oncology, v. 32, n. 5, p. 402-408, feb. 2014.

KRELL, J.; STEBBING, J. Broader implications of a stageguided stratified therapeutic approach for AIDS-related Kaposi's sarcoma. Journal of Clinical Oncology, v. 32, n. 5, p. 373-375, feb. 2014.

LEÃO, J. C. et al. Herpes vírus humano-8 e Sarcoma de Kaposi. Revista da Associação Médica Brasileira, v. 45, n. 1, p. 55-62, jan./mar. 1999.

LUNDGREN, J. D. Kaposi's sarcoma and its management in AIDS patients: Recommendations from a Scandinavian study group. Scandinavian Journal of Infectious Diseases, v. 29, n. 1, p. 3-12, 1997.

MEDURI, G. U. et al. Pulmonary Kaposi's sarcoma in the acquired immune deficiency syndrome: clinical, radiographic, and 
pathologic manifestations. The American Journal of Medicine, v. 81 , n. 1 , p. $11-18$, jul. 1986

NORTHFELT, D. W. et al. Efficacy of pegylated-liposomal doxorubicin in the treatment of AIDS-related Kaposi's sarcoma after failure of standard chemotherapy. Journal of Clinical Oncology, v. 15, n. 2, p. 653-659, feb. 1997.

PANTANOWITZ, L.; DEZUBE, B. J. Kaposi sarcoma in unusual locations. BMC Cancer, v. 8, n. 1, p. 1, jul. 2008.

PDQ ${ }^{\circledR}$ Adult Treatment Editorial Board. PDQ Kaposi Sarcoma Treatment. Bethesda, MD: National Cancer Institute, 2015. Disponível em: <http://www.cancer.gov/types/softtissue-sarcoma/hp/kaposi-treatment-pdq $>$. Acesso em $5 \mathrm{dez}$. 2016.
PORTSMOUTH, S. et al. A comparison of regimens based on non-nucleoside reverse transcriptase inhibitors or protease inhibitors in preventing Kaposi's sarcoma. Aids, v. 17, n. 11, p. F17-F22, jul. 2003.

RADU, O.; PANTANOWITZ, L. Kaposi Sarcoma. Archives of Pathology \& Laboratory Medicine, v. 137, n. 2, p. 289-294, 2013.

SCHWARTZ, R. A. Kaposi's sarcoma: an update. Journal of Surgical Oncology, v. 87, n. 3, p. 146-151, sep. 2004.

STEBBING, J.; PORTSMOUTH, S.; BOWER, M. Insights into the molecular biology and sero-epidemiology of Kaposi's sarcoma. Current Opinion in Infectious Diseases, v. 16, n. 1, p. 25-31, feb. 2003. 